\title{
The integration of operational AO software into the LBTO
}

\author{
Xianyu Zhang ${ }^{a}$, Christopher Biddick ${ }^{a}$, Guido Brusa Zappellini ${ }^{a}$, Julian Christou ${ }^{a}$, Luca Fini ${ }^{b}$, \\ Paul Grenz ${ }^{c}$, Stephen Hooper ${ }^{a}$, Douglas Miller ${ }^{a}$, Alfio Puglisi ${ }^{b}$, Fabio Rossi ${ }^{b}$, Douglas \\ Summers $^{a}$, Kellee Summers ${ }^{a}$, Gregory Taylor ${ }^{a}$, Marco Xompero ${ }^{b}$ \\ ${ }^{a}$ Large Binocular Telescope Observatory, University of Arizona, 933 N. Cherry Ave, Tucson, \\ AZ 85721, U.S.A.; \\ ${ }^{b}$ Osservatorio Astrofisico di Arcetri INAF, Largo Enrico Fermi, 5, 50125 Firenze FI, Italy; \\ ${ }^{c}$ The Large Binocular Telescope Interferometer, University of Arizona, 933 N. Cherry Ave, \\ Tucson, AZ 85721, U.S.A.
}

\begin{abstract}
After Arcetri team handover of the adaptive optics software to the Large Binocular Telescope Observatory this year, new functionalities and improvements will be implemented for daily operation and integration with the telescope. These include: safety alarm enhancements, web interface for display and control through INDI interface, operation interface upgrades, Zabbix and logging analysis/reporting improvements, and HDF5 based decimated telemetry for correlation with other Large Binocular Telescope Observatory telemetry. The adaptive optics system will be upgraded to Centos 7, and GUIs will be upgraded to Qt4. Finally, multiple disparate versions of AO supervisor software at LBTO, i.e, FLAO, UAO, SOUL, and LBTI, will be consolidated.
\end{abstract}

Keywords: Adaptive Optics, Large Binocular Telescope, Software, Adaptive Secondary Mirror, Operation

\section{INTRODUCTION}

The Large Binocular Telescope (LBT), operated by the he Large Binocular Telescope Observatory (LBTO), uses two $8.4 \mathrm{~m}$ apertures on a common mount to support independently or interferometrically observations. ${ }^{1}$ With its permanently mounted Adaptive Secondary Mirrors (ASM), the LBT is designed for adaptive optics (AO) observations. The ASM on the each side of the telescope has 672 voice-coil actuators, which makes the telescope achieves the $8.4 \mathrm{~m}$ diffractionlimited performance as two independent telescopes or achieves a $23 \mathrm{~m}$ baseline along one axis as an Fizeau and nulling interferometer.

All the AO systems on the LBT take the advantages of ASMs, but they are at different focal stations, as seen in the figure 1 . These $\mathrm{AO}$ systems cover a wide range of $\mathrm{AO}$ types ${ }^{2}{ }^{2}$ which include the single conjugated natural guide star adaptive optics system - First Light Adaptive Optics (FLAO) ${ }^{3}$ and Large Binocular Telescope Interferometer (LBTI) AO, ${ }^{4}$ the ground conjugated laser guide star system - Advanced Rayleigh Guided Ground Layer Adaptive Optics system (ARGOS), ${ }^{5}$ the multi-conjugate natural guide star adaptive optics system- LINCNIRVANA MCAO. ${ }^{6}$

Since the complexity and new technology, the ASMs were developed and tested in parallel as the LBT telescope. In order to support the ASMs related activities, the AO software are developed, but separated from the Telescope Control Software (TCS). After the ASMs were operated on the telescope, the operational AO software needs to be integrated into the LBT TCS system.

Further author information, send correspondence to Xianyu Zhang, E-mail: xzhang@lbto.org 


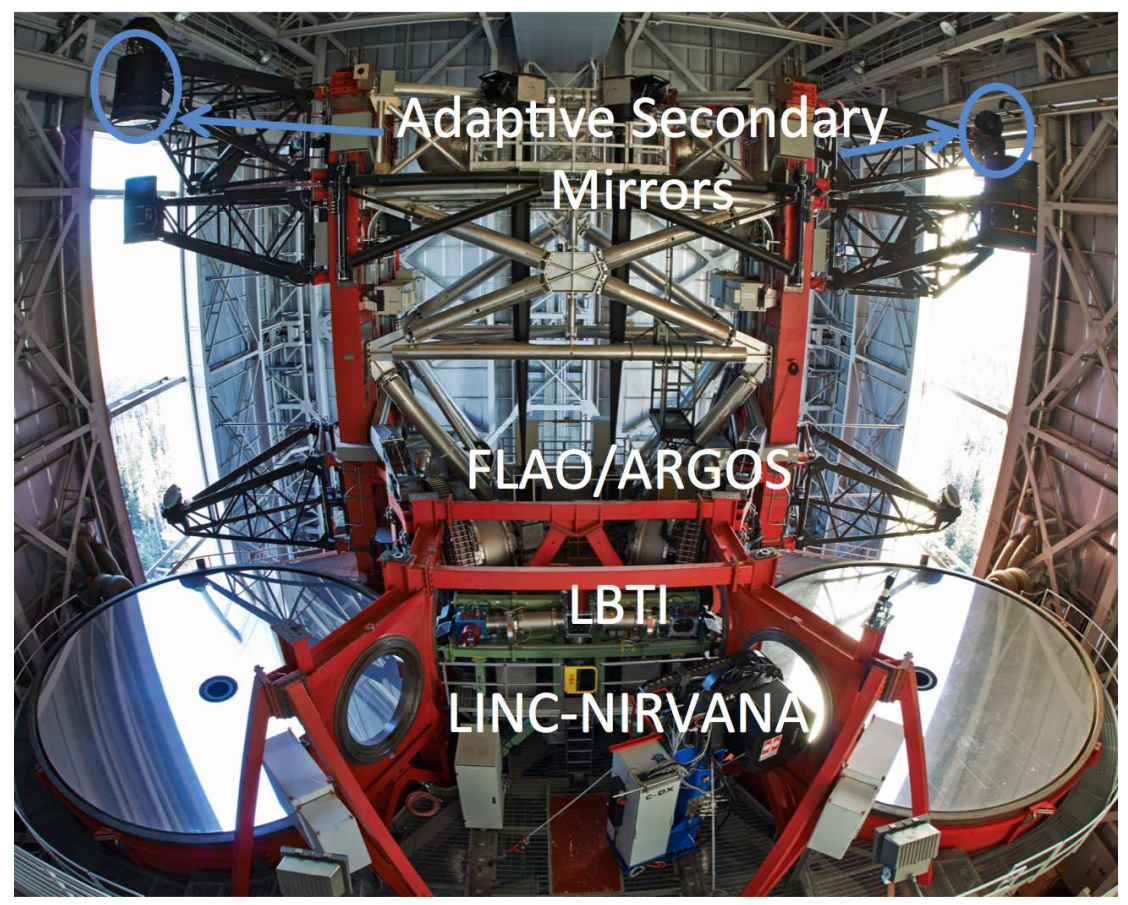

Figure 1. The adaptive secondary mirrors and their correspoing AO systems on the Large Binocular Telescope. As seen in the figure, the $\mathrm{AO}$ systems side at different focal stations, which are between the two $8.4 \mathrm{~m}$ primary mirrors. Note that this photograph was taken before the LINC-NIRVANA was installed. Photo by Enrico Sacchetti, 2014.

\section{FIRST LIGHT ADAPTIVE OPTICS SYSTEM}

The ASMs were developed and tested at the Arcetri Astrophysical Observatory, INAF, Italy, together with its modulated pyramid wavefront sensor, the First Light Adaptive Optics (FLAO) system was developed. ${ }^{3}$ As seen in figure 2, the FLAO software, also called AO supervisor, has two main computer boxes, the ADSEC and WFS. The ADSEC software talks and controls the ASMs, the WFS software is responsible for the WFS components . The WFS and ADSEC are communicated by the MsgD-RTDB, which is short for message daemon real-time database. On the high level, the wfsarb (WFS arbitrator) and adsecarb (ADSEC arbitrator) are used to manage each side. The aoarb (AO arbitrator), running on ADSEC, controls the wfsarb and the adsecarb, as well as communicates with the AOS system, which is inside the TCS system.

Thanks to the FLAO software structure design, all other AO systems software only need to communicate with the high level FLAO interface. Since the ASMs are used by all AO systems, the adsecarb/aoarb is used by all AO systems. The LBTI AO system has nearly same wavefront sensor as FLAO, the FLAO WFS software is also used by LBTI with few modifications.

\section{AO SOFTWARE AND TELESCOPE CONTROL SOFTWARE INTEGRATION}

Since the AO systems, especially the ASMs, are part of the telescope, it is crucial to let the FLAO software and TCS communicate with each other, all the time. In the case of non-AO seeing-limited operations, on the $\mathrm{AO}$ side, it is import to get the basic telescope information, like telescope elevation, swing arm status, etc, on the telescope TCS side, it is also import to get the ASM shell status, and inform telescope operation to make actions if needed. Obviously, in the AO operation case ${ }^{7}$ the telescope needs to know the AO system status, performance and activities, as well as control the AO system. The FLAO software, which is the interface for all the AO systems software, is need to be integrated into the telescope. 


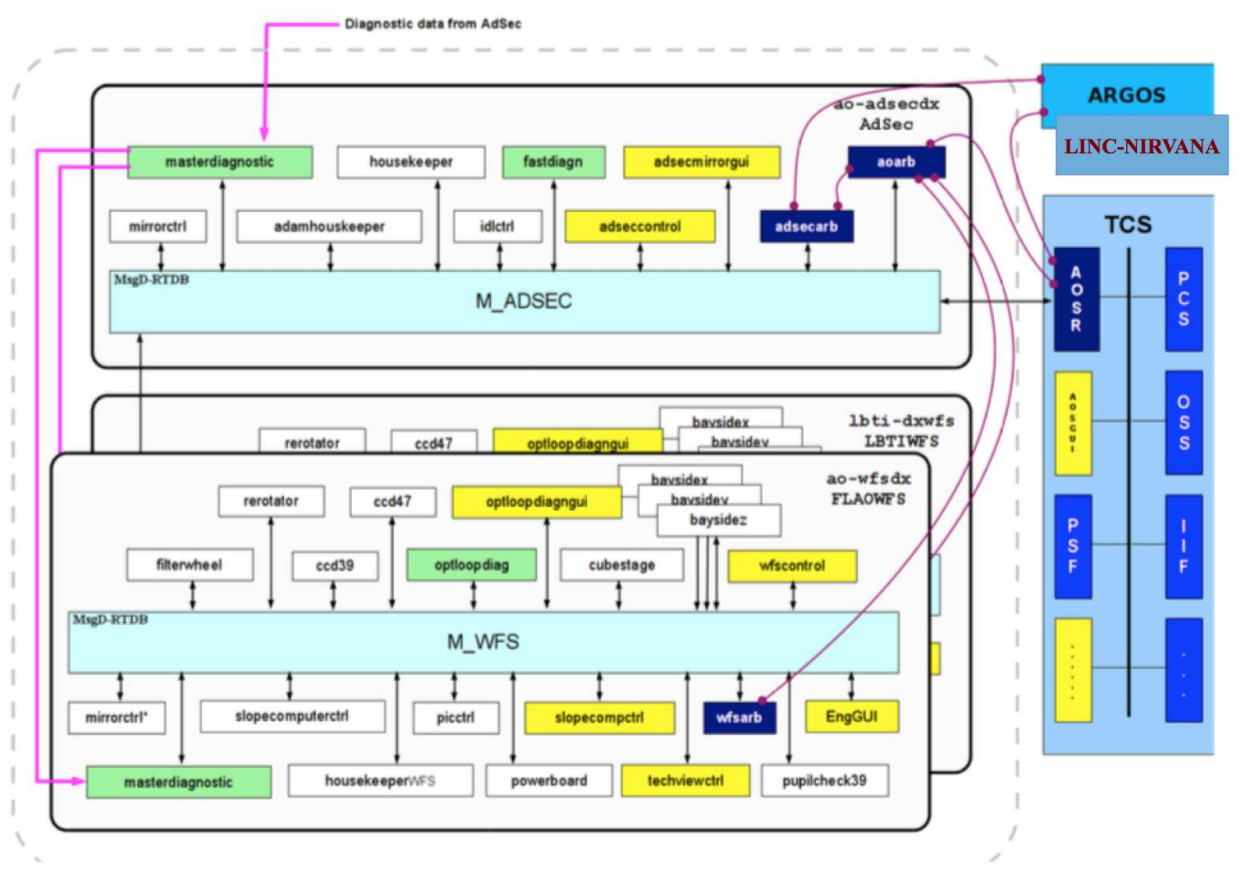

Figure 2. The first light adaptive optics software structure and its relations with the telescopes control software, and other AO systems software, i.e., LBTI, ARGOS and LINC-NIRVANA. Note, the right arm of the LBT (labeled as dx or R ) is used in the figure.

\subsection{FLAO Web interface}

In the LBTI AO system, the web interface based on the Instrument Neutral Distributed Interface (INDI) library ${ }^{8}$ was created. In order to give an easy and convenient access to the FLAO system, the LBTI AO INDI and web interface were modified for the FLAO system. The FLAO INDI servers are running on the ADSEC, WFS and LBTO web servers, the web browser runs as the frontends clients.

The FLAO web interface has two levels, the high and low level. Both levels are running in real-time. As seen in figure 3, the high level gives the basic AO and telescope states. The main users are the telescope operator and astronomer. As seen in figure 4, the low level interface, designed for the AO operator, gives detailed information about FLAO system, including the ASM shell shape, wavefront slopes, and acquisition camera image. The operator can use the low level interface to control the telescope and wavefront sensor, as shown in figure 4. As seen in figure 5, a bunches of the AO performance information is given, like the the wavefront slope RMS, ASM mirror commands RMS, Tip-Tilt RMS and so on. The level system status are also can be seen, as shown in figure 5. With the low level interface, the AO operator can easily remotely access the FLAO system to diagnostic and analysis the AO performance.

\subsection{Integrate AO Telemetry into Telescope Telemetry}

Aims to achieve highly efficient observatory operations by continuous evaluation and improvement of facility and instrumentation metrics, ${ }^{9,10}$ a suite of tools using in-house development and open source applications are developed to handle the telescope telemetry. As seen in figure 6, with the tools, the AO telemetry can be displayed through web-based two- and three-dimensional time series data visualization. The AO telemetry is transferred to TCS through AOS, as seen in figure 2. Then the AO telemetry is saved in HDF5 format and as part of the telescope telemetry.

To demonstrate the AO telemetry visualization, as shown in figure 6 , after choosing the data structure (Subsystems, Side, Stream and Variables) and time period, the telemetry display is seen in the left side of figure 


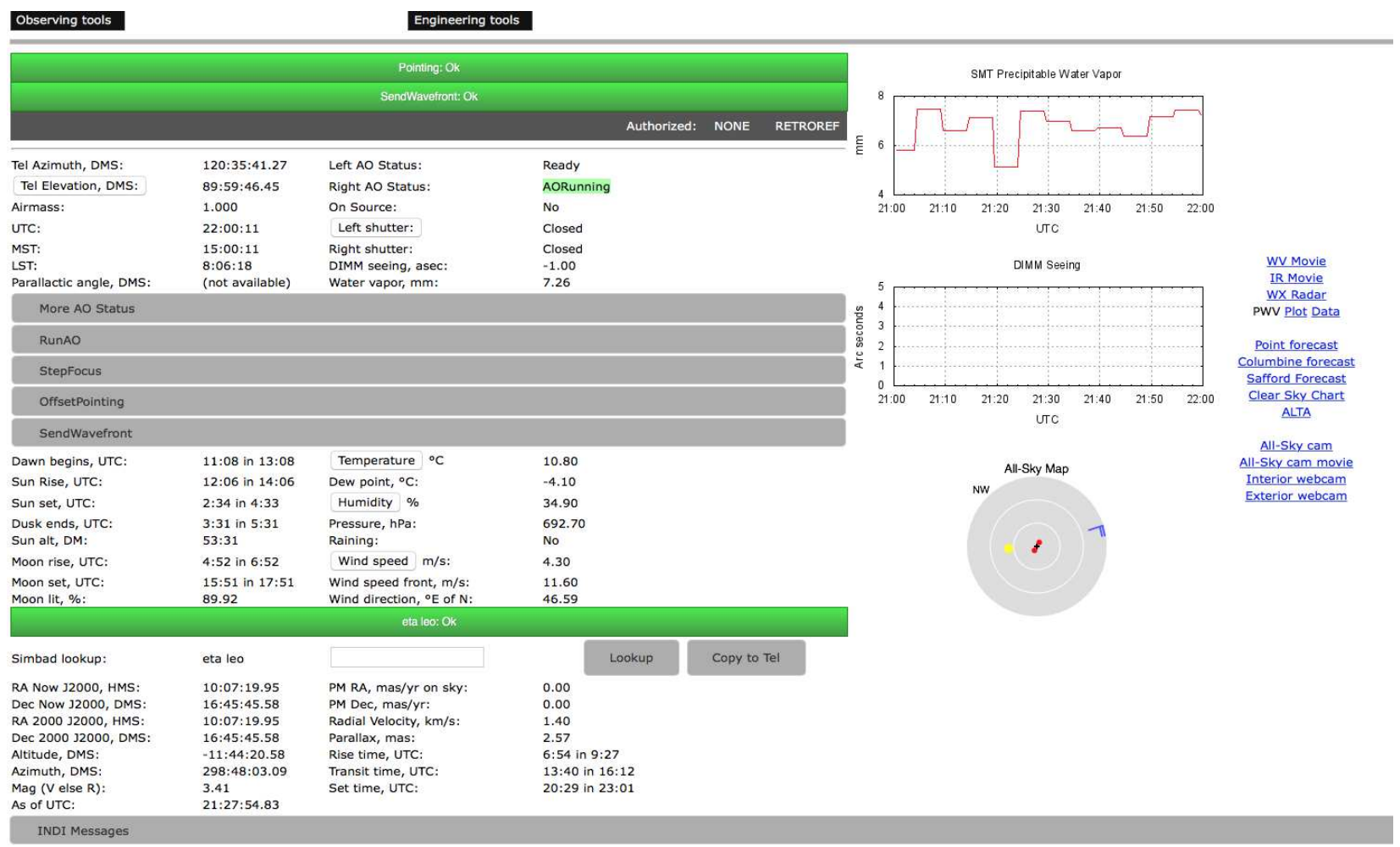

Figure 3. The AO and telescope real-time information though web interface.

7. A small time scale can be selected from the telemetry display, as seen in the right side of figure 7 , the dispaly is automatically changed to show the detailed information during that short time.

\subsection{Integrate AO Alarms into Telescope Alarm Handler}

To monitor telescope safety and inform the corresponding people on the telescope (especially the operator) with corresponding guidance and display, the alarm handler was introduced in the telescope TCS system. ${ }^{11}$ Since the ASMs are part of the telescope, it is important to include the AO/ASMs information in the alarm handler system. $^{12}$

The AO alarms included in the alarm handler are shown in Figure 8. Since the FLAO software should always be running and checking the ASM status, an AO script, monitored by the crontab job, is used to check the AO software running status and several critical critical ASM parameters, i.e.: TSS temperature, actuator average current, system cooling flow and telemetry status. The AO script sends the monitored parameters to the alarm handler and sends emails to the AO person when the parameters are outside the safe range.

As mentioned in section 3.2, the telemetry information is transferred from FLAO to the TCS, where the AO system status, i.e., ASM and AO subsystem status, telescope elevation and anemometer information, together with the ASM status, i.e., ASM main power, ASM Adam power, TSS status, are continuously monitored and sent to the alarm handler. Specific guidance actions to be taken are reported in the alarm handler GUI (figure 8) to instruct the telescope operator on how to handle each alarm case. This alarm handler system provides a compact and easy to use monitoring system, which improves the daily operation efficiency and system safety.

On the wavefront sensor side, the AO system writes the critical wavefront sensor parameters in the MySQL database. The Zabbix reads the records, makes the plot and send emails to the corresponding people when the some parameters are in alarm, like hardware high temperature. 


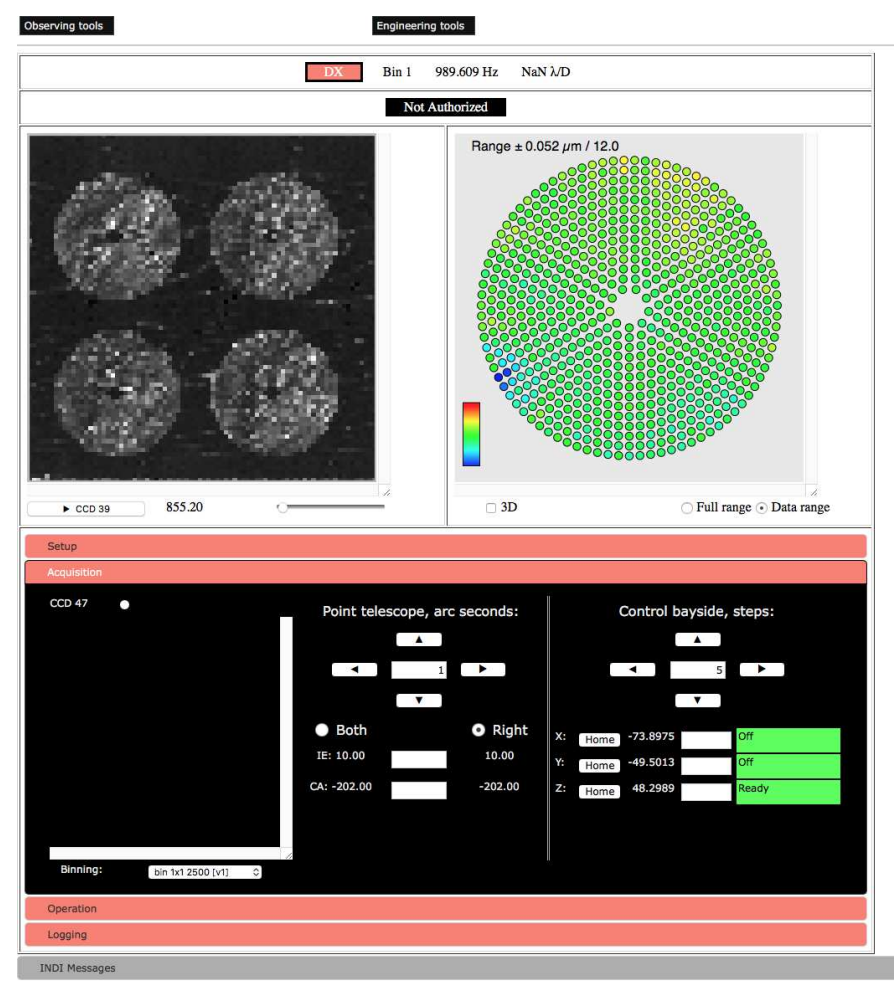

Figure 4. FLAO real-time display and control interface. The CCD39 wavefront sensor data is displayed in the top-left of the figure, the ASM shell shape is shown in the top-right of the figure. The CCD47 acquisition camera display, telescope pointing and adjustment, and wavefront sensor bayside stage control are shown in the lower parts of the figure.

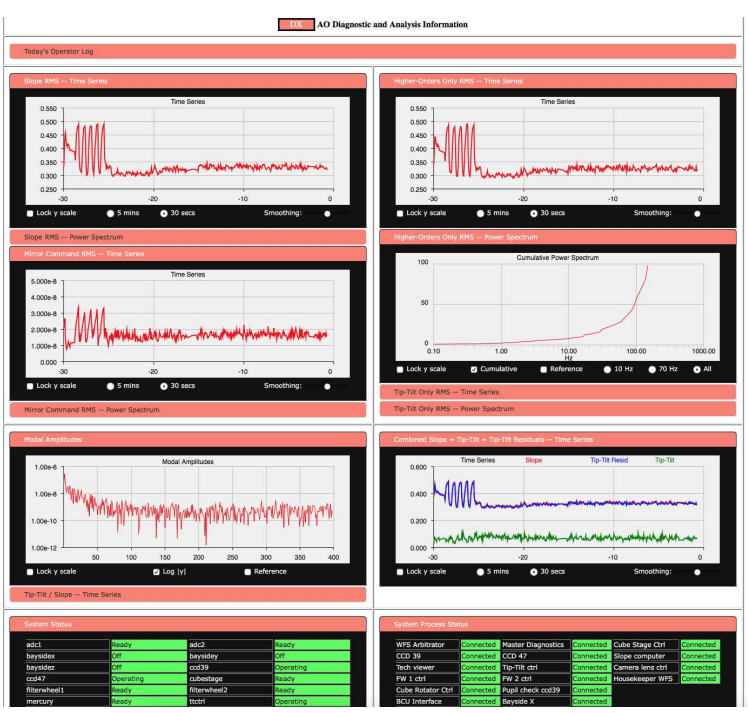

Figure 5. FLAO diagnostic and analysis information. The wavefront RMS, ASM commands RMS, Tip-Tilt and other parameters can be shown in different time scale. The low level system status with color can help the AO operator to notices the errors. 


\section{Telemetry Visualization}

Select subsystem(s) for one or two datasets. (Hint: use two datasets for correlations involving different streams). Then select side, stream, (optional) variable(s), desired date(s) and time filters. Note: Dates and Times are in UTC Need help graphing a CSV file? Go here: Graph CSV file

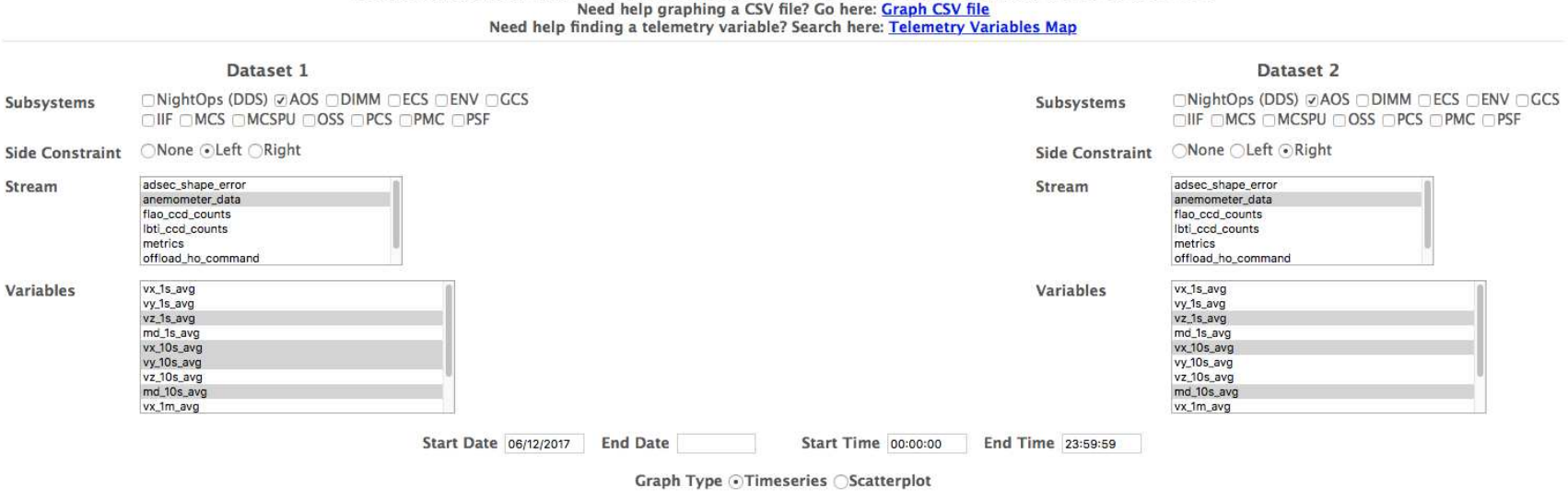

Figure 6. The AO telemetry integrated in the telescope telemetry system. Entry page for correlating and graphing any (reasonable) number of parameters from one or two telemetry streams on a single graph.

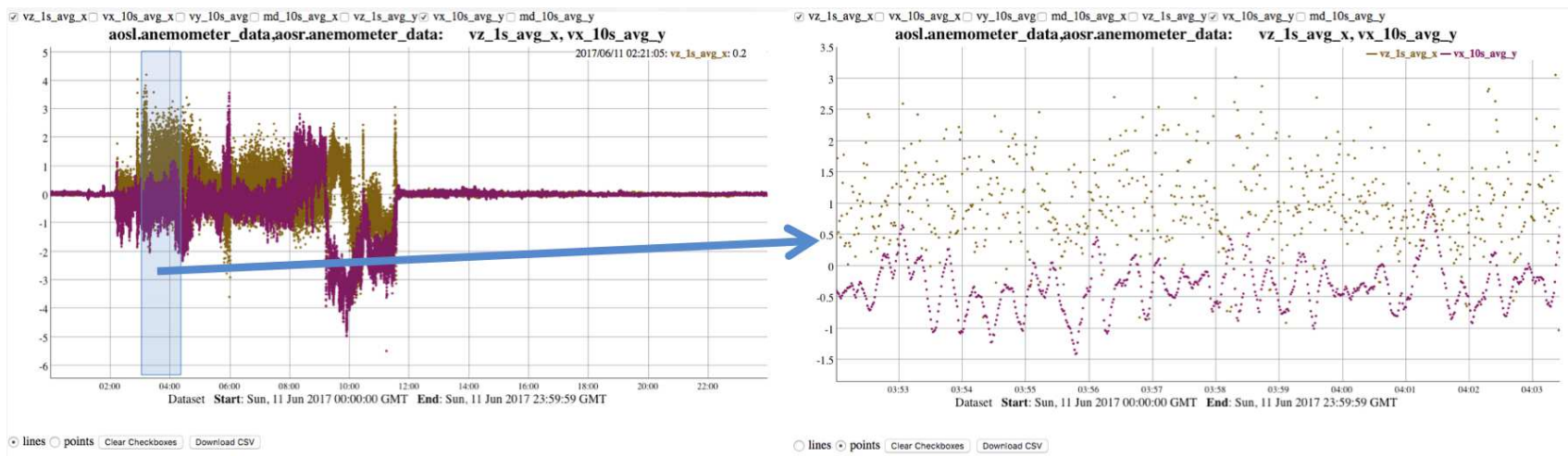

Figure 7. An demonstration of the AO telemetry visualization. The resulting display of the selection in figure 6 is shown in the left side of the figure. The zoom of the selected time period in the left figure is shown in the right side of the figure.

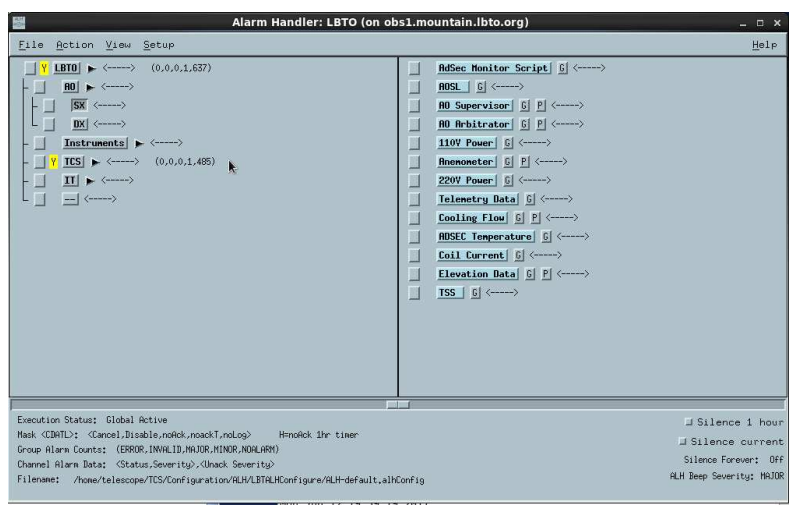

Figure 8. AO status and alarms in the LBTO alarm handler system. The P button invokes the GUIs on the AO boxes. The $\mathrm{G}$ button give the guidances. 


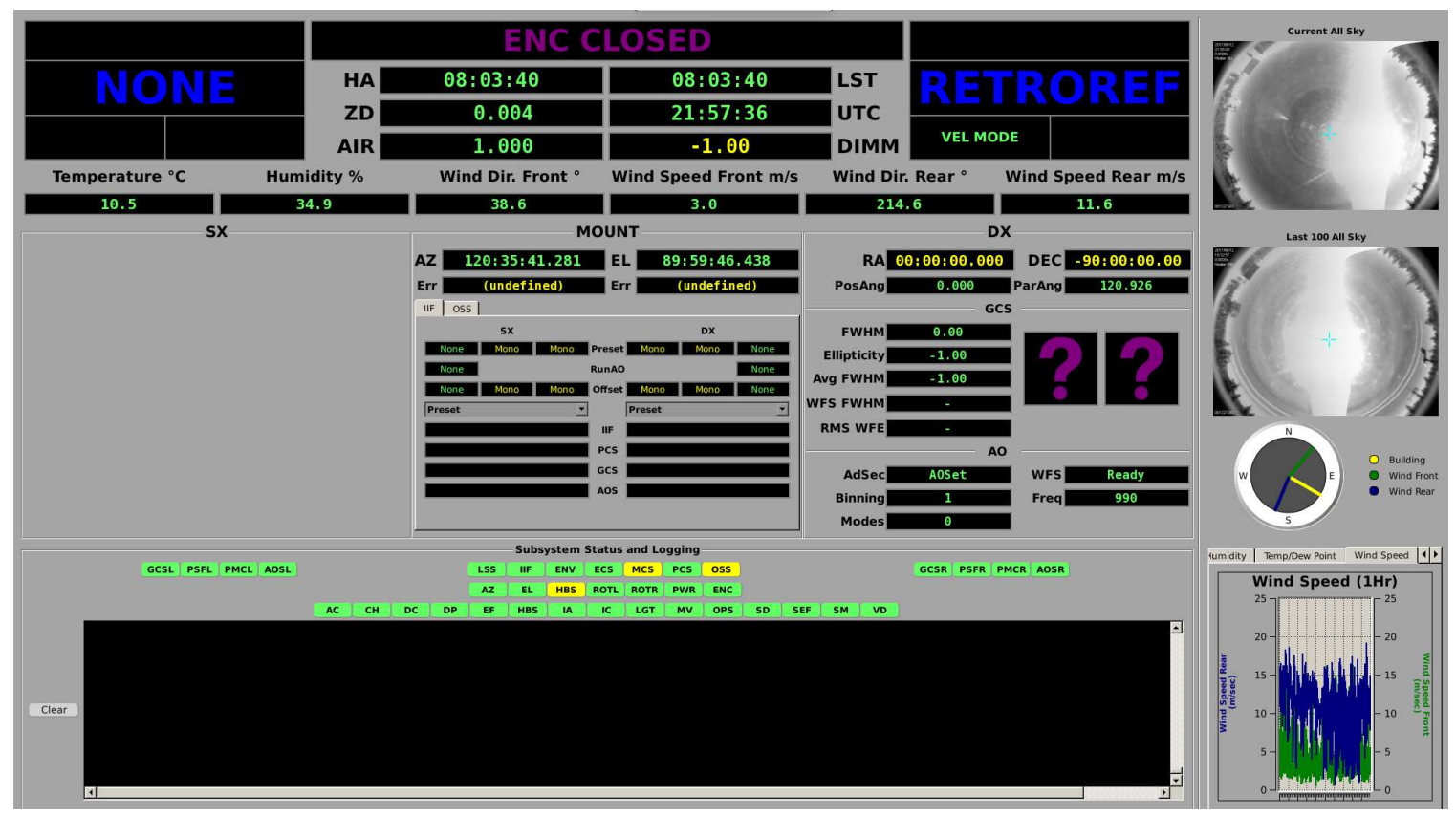

Figure 9. The FACSUM at the LBTO. The basic telescope information (dome open/close, elevation, RA, authorized instruments, etc), site environments (wind speed, seeing, humidity, and so on), TCS subsystems information, the AO system and its ASM information, are shown.

\subsection{Integrate AO Status on Telescope's FACSUM and Status Server}

To monitor telescope status and give the observer, telescope operator and management the live and easy accessible information about the telescope, its instruments and site environments, the FACSUM and status server web interface are given.

As seen in figure 9, when the AO system is being authorized/used, the basic AO system information is displayed. The FACSUM is displayed in the control room on the mountain and remote control room in Tucson. In the nighttime, the observer can get the live information about the $\mathrm{AO}$ system and make the corresponding actions and/or suggestions to the telescope operator if needed.

The status server provides the web interface to display the high-level telescope status, and shows their corresponding status in different color. As seen in figure 10, the AO systems (AO SX and AO DX) are integrated in the status server. The status server is designed to show high level information, the detailed information and guidance is not given, but can be founded in the alarm handler, as discussed in section 3.3.

\section{SUMMARY}

The LBT is designed as an AO fed telescope. With its 672-actuator ASMs on both sides, the LBT is capable of varies types of $\mathrm{AO}$ systems. In oder to improve the telescope and $\mathrm{AO}$ operation efficiency, performance and safety, the AO operation software, is integrated into the telescope control software. The AO system status and control interface can be accessed from the web interface. The AO system telemetry is recorded and analyzed as the LBT LBTO telemetry. The ASM safety alarms are integrated to the telescope alarm handler. The AO status is also displayed in the tlescope's FACSUM and status server. In the future, the FLAO system will be upgraded to Centos 7, and GUIs will be upgraded to Qt4. Later, multiple disparate versions of AO supervisor software at LBTO, i.e, FLAO, Unified Adaptive Optics (UAO), Single Conjugate Adaptive Optics Upgrade (SOUL), ${ }^{13}$ and LBTI, will be consolidated. 


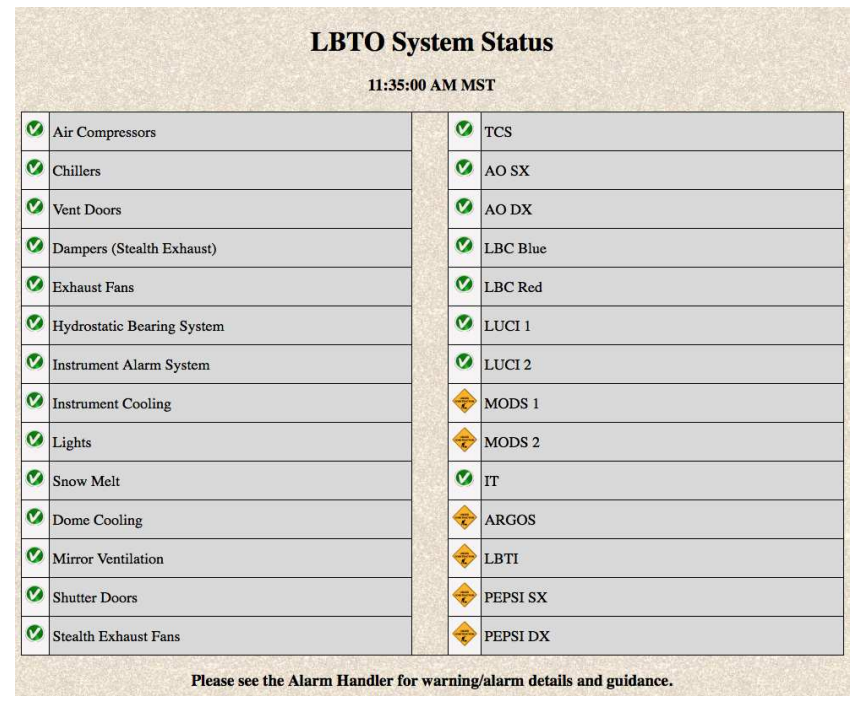

Figure 10. The status server web display at the LBTO.

\section{ACKNOWLEDGMENTS}

The LBT is an international collaboration among institutions in the United States, Italy and Germany. LBT Corporation partners are: The University of Arizona on behalf of the Arizona Board of Regents; Istituto Nazionale di Astrofisica, Italy; LBT Beteiligungsgesellschaft, Germany, representing the Max-Planck Society, The Leibniz Institute for Astrophysics Potsdam, and Heidelberg University; The Ohio State University, and The Research Corporation, on behalf of The University of Notre Dame, University of Minnesota and University of Virginia.

\section{REFERENCES}

[1] Hill, J. M., Ashby, D. S., Brynnel, J. G., Christou, J. C., Little, J. K., Summers, D. M., Veillet, C., and Wagner, R. M., "The Large Binocular Telescope: binocular all the time," in [Ground-based and Airborne Telescopes V], Proc. SPIE 9145, 914502 (July 2014).

[2] Christou, J. C., Brusa, G., Conrad, A., Esposito, S., Herbst, T., Hinz, P., Hill, J. M., Miller, D. L., Rabien, S., Rahmer, G., Taylor, G. E., Veillet, C., and Zhang, X., "Adaptive optics capabilities at the Large Binocular Telescope Observatory," in [Adaptive Optics Systems V], Proc. SPIE 9909, 99092E (July 2016).

[3] Tozzi, A., Stefanini, P., Pinna, E., and Esposito, S., "The double pyramid wavefront sensor for LBT," in [Adaptive Optics Systems], Proc. SPIE 7015, 701558 (July 2008).

[4] Hinz, P. M., Defrère, D., Skemer, A., Bailey, V., Stone, J., Spalding, E., Vaz, A., Pinna, E., Puglisi, A., Esposito, S., Montoya, M., Downey, E., Leisenring, J., Durney, O., Hoffmann, W., Hill, J., Millan-Gabet, R., Mennesson, B., Danchi, W., Morzinski, K., Grenz, P., Skrutskie, M., and Ertel, S., "Overview of LBTI: a multipurpose facility for high spatial resolution observations," in [Optical and Infrared Interferometry and Imaging V], Proc. SPIE 9907, 990704 (Aug. 2016).

[5] Raab, W., Rabien, S., Gaessler, W., Esposito, S., Antichi, J., Lloyd-Hart, M., Barl, L., Beckmann, U., Bonaglia, M., Borelli, J., Brynnel, J., Buschkamp, P., Busoni, L., Carbonaro, L., Christou, J., Connot, C., Davies, R., Deysenroth, M., Durney, O., Green, R., Gemperlein, H., Gasho, V., Haug, M., Hubbard, P., Ihle, S., Kulas, M., Loose, C., Lehmitz, M., Noenickx, J., Nussbaum, E., Orban De Xivry, G., Quirrenbach, A., Peter, D., Rahmer, G., Rademacher, M., Storm, J., Schwab, C., Vaitheeswaran, V., and Ziegleder, J., "Status of ARGOS - The Laser Guide Star System for the LBT," in [Proceedings of the Third AO4ELT Conference], Esposito, S. and Fini, L., eds., 106 (Dec. 2013).

[6] Herbst, T. M., Ragazzoni, R., Bertram, T., Bizenberger, P., Briegel, F., Hofferbert, R., and Kürster, M., "LINC-NIRVANA at LBT: final preparations for first light," in [Ground-based and Airborne Instrumentation for Astronomy VI], Proc. SPIE 9908, 99080N (Aug. 2016). 
[7] Miller, D. L., Taylor, G., Christou, J. C., Zhang, X., Brusa Zappellini, G., Rahmer, G., Lefebvre, M., Puglisi, A., Pinna, E., and Esposito, S., "Adaptive optics operations at the Large Binocular Telescope Observatory," in [Adaptive Optics Systems V], Proc. SPIE 9909, 99092G (July 2016).

[8] Downey, E. C., "Indi: Instrument-neutral distributed interface," in [http://www.clearskyinstitute.com/INDI/INDI.pdf], (June 2007).

[9] Summers, D. M., Biddick, C., Brynnel, J. G., Cox, C., De La Peña, M. D., Fisher, D., Golota, T., Hill, J. M., Hooper, S., Kraus, J., Sargent, T., Summers, K. R., and Veillet, C., "Large Binocular Telescope Observatory (LBTO) software and IT group operations status update and near-term development roadmap," in [Software and Cyberinfrastructure for Astronomy III], Proc. SPIE 9152, 915206 (July 2014).

[10] Summers, K. R., Summers, D. M., Biddick, C., and Hooper, S., "Telemetry correlation and visualization at the Large Binocular Telescope Observatory," in [Software and Cyberinfrastructure for Astronomy IV], Proc. SPIE 9913, $99131 \mathrm{Z}$ (Aug. 2016).

[11] De La Peña, M. D., Biddick, C., Summers, K., and Summers, D., "LBTO Alarm Notification/Management and Error Diagnostic Tools," in [Astronomical Data Analysis Software an Systems XXIV (ADASS XXIV)], Taylor, A. R. and Rosolowsky, E., eds., Astronomical Society of the Pacific Conference Series 495, 367 (Sept. 2015).

[12] Brusa, G., Ashby, D., Christou, J. C., Kern, J., Lefebvre, M., McMahon, T. J., Miller, D., Rahmer, G., Sosa, R., Taylor, G., Vogel, C., and Zhang, X., "Engineering aspects of the Large Binocular Telescope Observatory adaptive optics systems," in [Adaptive Optics Systems V], Proc. SPIE 9909, 990903 (July 2016).

[13] Pinna, E., Esposito, S., Hinz, P., Agapito, G., Bonaglia, M., Puglisi, A., Xompero, M., Riccardi, A., Briguglio, R., Arcidiacono, C., Carbonaro, L., Fini, L., Montoya, M., and Durney, O., "SOUL: the Single conjugated adaptive Optics Upgrade for LBT," in [Adaptive Optics Systems V], Proc. SPIE 9909, 99093V (July 2016). 